\title{
April 2014 Tucson Critical Care Journal Club: Early Goal-Directed Therapy
}

\author{
The ProCESS investigators. A randomized controlled trial of protocol- \\ based care for early septic shock. New Engl J Med. 2014 epub ahead of \\ print. Available at: http://www.neim.org/doi/full/10.1056/NEJMoa1401602 \\ (accessed 4/24/14).
}

In 2001, Early Goal Directed Therapy (EGDT) was demonstrated to improve 60 day mortality as compared to usual care practices, $44.3 \%$ vs. $56.9 \%$, respectively $(p=0.03)(1)$. EGDT and its components have since been incorporated into the major guidelines for the treatment of sepsis $(2,3)$. The subsequent critical care literature has focused on adherence to EGDT protocols, the value of individual protocol components, and alternative protocols that are less standardized (4-6). The ProCESS trial was conducted to assess the continued benefit of protocolbased sepsis treatment strategies in a contemporary setting.

ProCESS randomized 1351 adult patients who were suspected to have septic shock ( $\geq 2$ SIRS criteria plus refractory hypotension and/or elevated lactate) to the original EGDT protocol, a newer simplified protocol, or usual care. The study took place in 31 academic centers in the United States; these centers could not have been previously using septic shock treatment protocols. The simplified protocol differed from the original EGDT protocol by not mandating central venous monitoring of oxygen saturation (ScVO2) and by allowing greater flexibility of fluid, vasopressor, and transfusion treatment to achieve blood pressure and shock index goals.

The primary outcome was the difference in 60 day mortality between the two protocol-based approaches and usual care. If this comparison was significant, then the two protocol-based approaches would be compared. The initial sample size calculation estimated that 1950 patients would be needed to achieve $80 \%$ power to detect a 6-7\% absolute reduction in mortality assuming that mortality would be $30-46 \%$ in the usual care group. Because mortality in the usual care group was observed to be lower than expected $(20 \%)$ a smaller sample size was deemed sufficient to detect the same absolute risk reduction without loss of power.

Of the 12,707 patients who were screened, 1,351 (10\%) were ultimately enrolled. Major reasons for exclusion included lack of hypoperfusion (30\%), lack of refractory hypotension (26\%), study logistic issues $(13 \%)$, no suspected infection $(11 \%),<2$ SIRS criteria $(7 \%)$, or a Do-Not-Resuscitate order $(7 \%)$.

There was no statistical difference in 60 day mortality between the two protocolbased strategies and usual care, $19.5 \%$ vs. $18.9 \%$, respectively $(p=0.83)$. Among secondary endpoints, the simplified protocol was associated with a greater risk of renal replacement therapy as compared to the other two groups, but this association was marginally significant $(p=0.04)$. Admission to the ICU 
was more likely in the original EGDT protocol $(91.3 \%)$ versus either the simplified protocol $(85.4 \%)$ or usual care $(86.2 \%, p=0.01)$. Serious adverse events were rare and no different across the 3 treatment groups.

There were significant differences observed across the original EGDT protocol, simplified protocol, and usual care groups with respect to the use of CVC $(93.6 \%$ vs. $56.5 \%$ vs. $57.9 \%$,respectively; $p=<0.001$ ), fluid administration within first 6 hours $(2.8 \mathrm{~L}$ vs. $3.3 \mathrm{~L}$ vs. $2.3 \mathrm{~L}$, respectively; $p=<0.001)$, vasopressor use $(54.9 \%$ vs. $52.2 \%$ vs. $44.1 \%$, respectively; $p=0.003)$, dobutamine use $(8 \%$ vs. $1.1 \%$ vs. $0.9 \%$, respectively; $p=0.001)$ and $\mathrm{PRBC}$ use $(14.4 \%$ vs. $8.3 \%$ vs. $7.5 \%$, respectively; $p=0.001$ ).

It is important to compare the mortality observed in the current study's usual care group with that observed in the 2001 study's usual care group, $18.9 \%$ vs. $56.9 \%$, respectively. This difference was observed despite similarities between the current study population and the 2001 study population: APACHE 2 score $(20.8$ vs. 20.4 , respectively), mean serum lactate level $(4.8 \mathrm{mmol} / \mathrm{L}$ vs. $6.9 \mathrm{mmol} / \mathrm{L}$, respectively), and age (62 vs. $67 \mathrm{yrs}$, respectively).

Given the advances in sepsis care that have occurred since 2001, it is difficult to interpret the current study's results. Taken at face value, they seem to suggest that protocol-based treatment is of no benefit. However, the importance of early sepsis recognition, aggressive fluid resuscitation, and timely antibiotic administration has become widely known and has been codified by professional societies and treatment guidelines. So much so that the treatment received in the usual care arm was difficult to distinguish from that received in the EGDT group with the exception of less central venous catheterization.

In summary, ProCESS did not demonstrate any benefit from protocolized treatment of septic shock; however, we cannot help but question the utility of this result given the apparent ubiquity of EGDT as routine practice in these academic medical centers.

Bhupinder Natt MBBS, Cristine Berry MD, Christian Bime MD and Joe Gerald PhD

University of Arizona

Tucson, AZ

\section{References}

1. Rivers E, Nguyen B, Havstad S, et al. Early goal-directed therapy in the treatment of severe sepsis and septic shock. N Engl J Med 2001; 345:136877. [CrossRef] [PubMed]

2. Dellinger RP, Carlet JM, Masur H, et al. Surviving Sepsis Campaign guidelines for management of severe sepsis and septic shock. Crit Care Med 2004; 32:858-73. [CrossRef] [PubMed] 
3. Dellinger RP, Levy MM, Carlet JM, et al. Surviving Sepsis Campaign: international guidelines for management of severe sepsis and septic shock: 2008. Crit Care Med 2008; 36:296-327. [CrossRef] [PubMed]

4. Carlbom DJ, Rubenfeld GD. Barriers to implementing protocol-based sepsis resuscitation in the emergency department- results of a national survey. Crit Care Med 2007; 35:2525-32. [CrossRef] [PubMed]

5. Jones AE, Shapiro NI, Roshon M. Implementing early goal-directed therapy in the emergency setting: the challenges and experiences of translating research innovations into clinical reality in academic and community settings. Acad Emerg Med 2007; 14:1072-8. [CrossRef] [PubMed]

6. Reade MC, Huang DT, Bell D, et al. Variability in management of early severe sepsis. Emerg Med J 2010; 27:110-5. [CrossRef] [PubMed] 